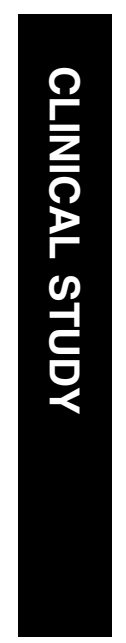

\section{The efficacy of} intravenous

ketorolac for pain relief in single-stage adjustable strabismus surgery: a prospective, randomized, placebo-controlled trial

${ }^{1}$ The Institute of Vision Research, Department of Ophthalmology, Yonsei University College of Medicine, Seoul, Korea

${ }^{2}$ Department of Ophthalmology, Kwandong University College of Medicine, Goyang, Korea

${ }^{3}$ Department of Ophthalmology, Soonchunhyang University College of Medicine, Bucheon, Korea

${ }^{4}$ Department of Anesthesiology and Pain Medicine, Yonsei University College of Medicine, Seoul, Korea

Correspondence: JB Lee, Institute of Vision Research, Department of Ophthalmology, Yonsei University College of Medicine,

250 Seongsanno,

Seodaemun-gu,

Seoul 120-752.

Korea

Tel: + 8222228 3570;

Fax: + 8223120541 .

E-mail: 491209@yuhs.ac

Received: 11 May 2010 Accepted in revised form: 20 September 2010: Published online: 19 November 2010

\begin{abstract}
Purpose To determine the efficacy of preoperative intravenous ketorolac in reducing intraoperative and postoperative pain and improving patient satisfaction in patients undergoing single-stage adjustable strabismus surgery.

Methods A prospective, randomized, placebo-controlled clinical trial was performed with 67 patients who underwent horizontal recti muscle surgery with adjustable sutures. The test group received intravenous ketorolac $(60 \mathrm{mg})$ before surgery, and the control group received intravenous normal saline. Topical $0.5 \%$ proparacaine was administered to both groups during surgery. Vital signs including heart rate and blood pressure were recorded every $10 \mathrm{~min}$ throughout the surgery. The patients were asked to rate their maximum intraoperative and postoperative pain scores using a numerical pain rating scale. Patient satisfaction was also assessed using a five-point analogue scale.

Results The ketorolac-premedicated patients had less pain both during and after surgery ( $P=0.033$ and $P=0.024$, respectively). There were no differences in vital signs during surgery and patient satisfaction between the two groups. Conclusions Intravenous ketorolac, when administered preoperatively for single-stage adjustable strabismus surgery under topical anaesthesia, was effective in reducing pain during and after surgery.
\end{abstract}

S Rhiu', SA Chung ${ }^{2}$, WK Kim¹, JH Chang ${ }^{3}$, SJ $\mathrm{Bae}^{4}$ and JB Lee ${ }^{1}$
Eye (2011) 25, 154-160; doi:10.1038/eye.2010.168; published online 19 November 2010

Keywords: one-stage adjustable strabismus surgery; topical strabismus surgery; ketorolac; eye pain; prospective; randomized trial

\section{Introduction}

Strabismus surgery aims to achieve precise ocular alignment and can be performed either traditionally (non-adjustable) or with adjustable sutures. In traditional strabismus surgery, one cannot alter the results once the surgery is complete, often yielding unpredictable results. In an effort to reduce this problem, adjustable suture surgery was described and popularised by Jampolsky in $1965 .^{1}$

The use of adjustable sutures in strabismus surgery has decreased the re-operation rate to $<10 \%$ (vs $20 \%$ with traditional strabismus surgery). ${ }^{2}$ Adjustable suture surgery also reduces the systemic risk to the patient as it is done under local anaesthesia, making postoperative recovery shorter and easier. ${ }^{2}$

Adjustable suture surgery is usually carried out in two stages; in the first stage, surgery is performed under general or regional anaesthesia. Once the effects of the anaesthesia dissipate, the second stage adjustment can be performed. ${ }^{3}$ The adjustable procedure is tricky for both surgeons and patients, as it is usually performed outside the operating room, and 
carries an increased risk of infection. Furthermore, the surgeon must wait for the anaesthetic effects to diminish, often causing patients to experience prolonged anxiety during this waiting stage. Single-stage adjustable suture surgery eliminates these problems. The ability to perform adjustments in the operating room during the surgery is more attractive with regard to patient monitoring, sterility, comfort, and timing. ${ }^{4}$ Additionally, the stability of one-stage adjustable sutures has been reported to be comparable to that of more widely used two-stage adjustable sutures. ${ }^{3,5,6}$

One drawback of single-stage adjustable suture surgery under topical anaesthesia is that patients typically experience more pain and discomfort during surgery, which leads to increased stress for both the surgeon and the patient. ${ }^{3}$ Patient compliance is essential during adjustable suture surgery, thereby making effective pain control a top priority.

Ketorolac tromethamine is a non-narcotic analgesic that inhibits cyclooxygenase activity in the arachidonic acid cascade and interferes with prostaglandin

production. Ketorolac is a non-steroidal anti-inflammatory drug (NSAID) with a $6 \mathrm{~h}$ half-life. ${ }^{7}$ It has been reported to have fewer side effects than narcotic analgesics, resulting in notably less sedation, nausea, and respiratory depression. ${ }^{8-10}$ These properties make ketorolac a potential solution for pain control in patients undergoing strabismus surgery. The effect of ketorolac has been evaluated in strabismus surgery; however, in these studies, the patients were under general or periocular anaesthesia. ${ }^{11-13}$

We prospectively evaluated the efficacy of preoperative intravenous ketorolac in reducing intraoperative and postoperative pain and in improving patient satisfaction in patients undergoing one-stage adjustable strabismus surgery.

\section{Materials and methods}

This study was conducted prospectively from November 2008 to September 2009. A total of 67 cases (greater than 10 years of age) of horizontal strabismus were enroled. Patients with restrictive strabismus, myasthenia gravis, paralytic strabismus, thyroid eye disease, or previous retinal detachment surgery were excluded. Basic haematology (haemoglobin, erythrocytes, white blood cells, and platelets), prothrombin time, activated partial thromboplastin time, biochemistry (total protein, urea, creatinine, glucose, electrolytes, aspartate transaminase, and alanine transaminase), urine analysis, chest $\mathrm{X}$-ray, and electrocardiogram (EKG) was screened preoperatively in all patients and patients who showed any abnormal results were excluded. Patients with obvious contraindications to ketorolac such as asthma, renal dysfunction, peptic ulceration, bleeding disorders, cardiovascular disease, mental retardation, or allergy to ketorolac or NSAIDS were also excluded.

The angle of deviation was measured using the alternate prism cover test or the Krimsky corneal reflex method. Manifest refraction and fundus examinations were also performed in all patients.

Patients were randomly divided into two groups with comparable ages, gender ratios, and types of surgery.

Patients in group 1 received intravenous saline placebo and patients in group 2 received intravenous ketorolac $(60 \mathrm{mg}) 30 \mathrm{~min}$ before surgery. One examiner received the treatment group assignment at the time of surgery and administered the study doses. All other personnel involved in the surgeries were masked as to the identity of the treatment groups. All patients were monitored intraoperatively for heart rate, continuous EKG, non-invasive blood pressure (NIBP), and oxygen saturation by pulse-oximeter. Another blinded examiner (WKK) assessed the maximum intraoperative and postoperative pain scores. Patient satisfaction was assessed $10 \mathrm{~min}$ following the completion of surgery.

In all patients, topical $0.5 \%$ proparacaine (Alcaine, Alcon-Couvreur, Puurs, Belgium) was instilled $10 \mathrm{~min}$ before and at the beginning of surgery in each eye and was additionally administered during the incisions of the conjunctiva and Tenon's capsule and also if the patient complained of discomfort during the surgery. The surgical techniques used in all patients included unilateral or bilateral recession or resection of the horizontal recti muscles with a limbal conjunctival incision. When surgery was performed on two muscles, the first muscle was operated on according to a preoperative estimation using conventional techniques. When the first phase of the surgery was completed, the patient was asked to sit upright on the operative table for adjustment. For patients who required spectacles, the spectacles were gas-sterilised, and an assistant held the frame of the spectacles in front of the patient during the examination in order to maintain sterile conditions. If the ocular alignment was theoretically correct and diplopia was not noted, the strabismus surgery was considered complete. If strabismus persisted, surgery was performed on the second muscle with an adjustable suture. All adjustable suture operations were performed with 6-0 Vicryl using a single-loop bow tie (the 'bow tie' technique). ${ }^{14}$ This knot consisted of a single overhand knot followed by a second overhand knot which was left in a single loop. This single-loop bow tie could be untied in order to adjust the position of the muscle. Ocular alignment was measured with the alternate prism cover test using a suitable fixation target for near and distance, while the patient was 
in the sitting position. The Krimsky prism reflex method was used in patients with sensory strabismus. The adjustment phase was repeated until the theoretical correct position was achieved and no diplopia was noted in the primary gaze position. After the muscle was appropriately adjusted, the bow was tied off by first cutting the loop and then carefully withdrawing the cut suture, leaving the knot in place. The square knot remained in place, joining the pole suture. Finally, the conjunctiva was closed with 6-0 black silk.

A good result was considered an angle of deviation of < eight prism dioptres with an absence of diplopia.

For data analysis, SPSS statistical software (version 15.0; SPSS Inc., Chicago, IL, USA) was used. The $\chi^{2}$-test was used for qualitative data including sex, diagnosis of strabismus (exodeviation $v$ s esodeviation), and type of surgery (recession only $v$ s resection included).

Comparison of continuous data including age, angle of deviation, and total duration of surgery was performed using the independent two-sample $t$-test. Repeated measure ANOVA was used for analysing heart rate, systolic NIBP, and diastolic NIBP recorded every $10 \mathrm{~min}$ during surgery. Multiple regression analysis with adjustments for age, sex, and type of surgery was used for comparing maximum intraoperative and postoperative pain scores and patient satisfaction scores. In this study, $P$-value $<0.05$ was considered statistically significant.

We certify that all applicable institutional and governmental regulations concerning the ethical use of human volunteers were followed during this research.

\section{Results}

During an 11-month period, 67 patients who underwent one-stage adjustable strabismus surgery performed by the same surgeon (JBL) met the study inclusion criteria and were randomized into the study (31 into the placebo group and 36 into the ketorolac premedicated group). There were no differences in patient characteristics, preoperative angle of deviation, duration of surgery, and type of surgery between the two groups (Table 1).

There were no differences in systolic blood pressure, diastolic blood pressure, (Figure 1) or pulse rate (Figure 2) during surgery between and within the two groups ( $P=1.00,0.70$, and 0.37 , respectively).

The patients premedicated with ketorolac had significantly less pain both during and after surgery ( $P=0.033$ and 0.024 , respectively). Patient satisfaction showed no difference between the two groups (Table 2).

None of the patients exhibited any undesirable side effects related to ketorolac.
Table 1 Basic clinical characteristics of the patients

\begin{tabular}{lccc}
\hline & $\begin{array}{c}\text { Placebo } \\
(\mathrm{n}=31)\end{array}$ & $\begin{array}{c}\text { Ketorolac } \\
(\mathrm{n}=36)\end{array}$ & P-value \\
\hline Age (years) & $20.84 \pm 11.09$ & $21.28 \pm 14.04$ & $0.102^{\mathrm{a}}$ \\
Sex & & & $0.202^{\mathrm{b}}$ \\
$\quad$ Male & $16(51.61 \%)$ & $13(36.11 \%)$ & \\
$\quad$ Female & $15(48.39 \%)$ & $23(63.89 \%)$ & \\
$\begin{array}{l}\text { Duration of surgery } \\
\text { (minutes) }\end{array}$ & $31.71 \pm 8.14$ & $29.72 \pm 9.71$ & $0.372^{\mathrm{a}}$ \\
& & & \\
$\begin{array}{l}\text { Diagnosis } \\
\quad \text { Exodeviation }\end{array}$ & $26(83.9 \%)$ & $30(83.3 \%)$ & $0.953^{\mathrm{b}}$ \\
$\quad$ Esodeviation & $5(16.1 \%)$ & $6(16.7 \%)$ & \\
& $31.0 \pm 9.81$ & $32.14 \pm 16.31$ & $0.683^{\mathrm{a}}$ \\
$\begin{array}{l}\text { Preop deviation (PD) } \\
\text { Surgery performed }\end{array}$ & & & $0.581^{\mathrm{b}}$ \\
$\quad \begin{array}{l}\text { Recession only } \\
\text { Resection included }\end{array}$ & $6(19.35 \%)$ & $9(25.00 \%)$ & \\
\hline
\end{tabular}

Abbreviations: Preop, preoperative; PD, prism dioptres.

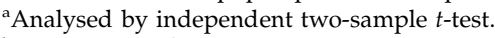

${ }^{\mathrm{b}}$ Analysed by $\chi^{2}$-test.

Data presented as mean \pm SD or absolute numbers (\%).

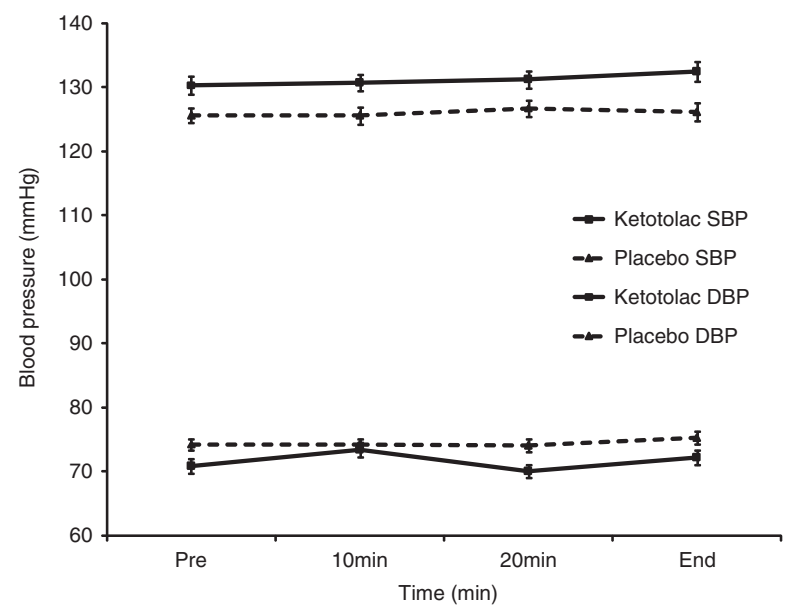

Figure 1 Comparison of blood pressures between the ketorolac and placebo groups. DBP, diastolic blood pressure; End, parameters measured at the conclusion of surgery; Pre, parameters measured at the beginning of surgery; SBP, systolic blood pressure; $10 \mathrm{~min}$, parameters measured $10 \mathrm{~min}$ after initiation of surgery; $20 \mathrm{~min}$, parameters measured $20 \mathrm{~min}$ after initiation of surgery.

\section{Discussion}

Adjustable suture strabismus surgery is thought to result in better ocular alignment and long-term stability compared with those of non-adjustable surgery. ${ }^{2}$ Single-stage adjustable strabismus surgery involves adjustments during the operative period; in contrast, two-stage adjustable strabismus surgery involves 


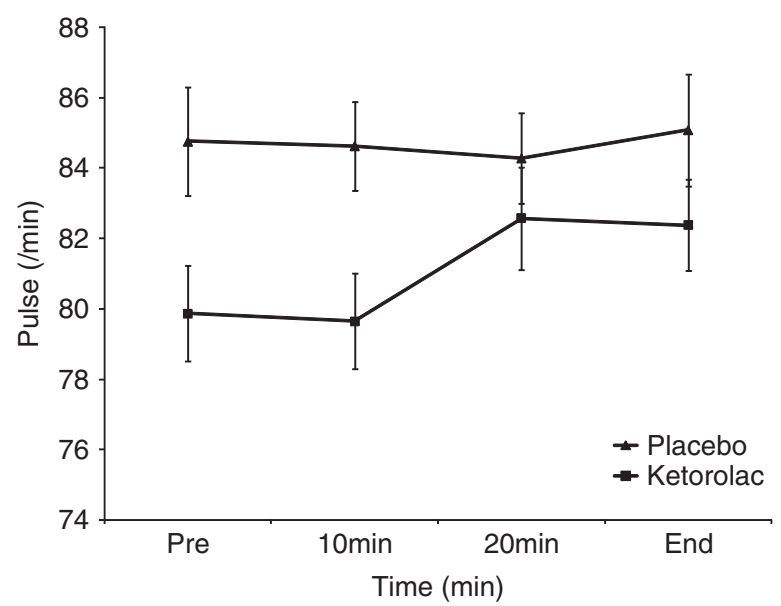

Figure 2 Comparison of pulse rates between the ketorolac and placebo groups. End, parameters measured at the conclusion of surgery; Pre, parameters measured at the beginning of surgery; $10 \mathrm{~min}$, parameters measured $10 \mathrm{~min}$ after initiation of surgery; $20 \mathrm{~min}$, parameters measured $20 \mathrm{~min}$ after initiation of surgery.

Table 2 Comparison of pain and patient satisfactions between the ketorolac and placebo groups

\begin{tabular}{lccc}
\hline & $\begin{array}{c}\text { Placebo } \\
(\mathrm{n}=31)\end{array}$ & $\begin{array}{c}\text { Ketorolac } \\
(\mathrm{n}=36)\end{array}$ & P-value $^{\mathrm{a}}$ \\
\hline Maximal intraop pain & $3.21 \pm 1.29$ & $2.76 \pm 1.21$ & 0.033 \\
Postoperative pain & $0.55 \pm 0.96$ & $0.13 \pm 0.37$ & 0.024 \\
Patient satisfaction & $4.06 \pm 0.63$ & $4.06 \pm 0.53$ & 0.640 \\
\hline
\end{tabular}

Abbreviation: Intraop, intraoperative.

${ }^{\mathrm{a}}$ Multiple regression analysis with adjustments for age/sex/surgery type. Data presented as mean \pm SD.

surgery performed under local or general anaesthesia followed by an adjustment $6-24 \mathrm{~h}$ later. ${ }^{15}$ The adjustable suture technique requires a fully awake and cooperative patient, recovery from anaesthesia, and return of muscle function. ${ }^{2}$ A certain period of time is required for full recovery when peribulbar, retrobulbar, or general anaesthesia is used for strabismus surgery. This increases the time and cost of the hospital stay and increases the risk of complications including retrobulbar haemorrhage, eyeball perforation, optic nerve injury, and systemic complications that may occur following general anaesthesia. Despite these risks, single-stage adjustable surgery is known to be a safe procedure. ${ }^{6,15-19}$ The success rate of single-stage adjustable surgery is comparable to that observed in previous studies of two-stage adjustment surgery performed under general or retrobulbar anaesthesia..$^{5,20,21}$ The primary disadvantages of single-stage adjustable surgery under topical anaesthesia include a longer procedure time and more intraoperative pain and discomfort of the patient. ${ }^{15,22}$
In order to decrease pain and patient discomfort, we evaluated the effect of a single dose of intravenous ketorolac on intraoperative and postoperative pain during single-stage adjustable surgery in a prospective, randomized, placebo-controlled trial.

Ketorolac is a NSAID with an analgesic effect that becomes effective $30 \mathrm{~min}$ after dosing and reaches a maximum effect at $2 h{ }^{23}$ The duration of strabismus surgeries is often short, and it would presumably be more effective to administer ketorolac before the surgical incision instead of at the completion of surgery. Ketorolac has proven to be effective in controlling perioperative pain and postoperative vomiting and nausea in strabismus and vitreoretinal surgeries..$^{8-13,23-28}$ As narcotics can cause excessive sedation in patients, resulting in respiratory depression and delayed discharge, ketorolac is preferred in the prevention and treatment of postoperative pain in strabismus patients.

Mendel et $a l^{11}$ reported that ketorolac was more effective in reducing postoperative vomiting in children undergoing strabismus surgery. Although postoperative pain scores did not differ significantly between the placebo and ketorolac groups, the scores in the ketorolac group tended to be lower. However, this study evaluated adjuvant ketorolac in childhood strabismus surgery performed under general anaesthesia. As such, it was impossible to accurately evaluate intraoperative and postoperative pain scores in preverbal children. Studies comparing the effects of intravenous ketorolac to those of pethidine ${ }^{27}$ and morphine ${ }^{13}$ also showed that the efficacy of ketorolac was comparable to those of opioids; however, these studies had limitations similar to that of the study by Mendel et al. ${ }^{11}$

Morrison $e t a l^{12}$ reported that intravenous ketorolac given at the conclusion of surgery was more effective than either oral acetaminophen or oral ibuprofen given 30-45 min after strabismus surgery in controlling postoperative pain. In their study, the patients were older than 12 years of age and surgery was performed under either general inhalational anaesthesia or local periocular lidocaine anaesthesia.

In the present study, we evaluated not only postoperative pain but also intraoperative pain during single-stage adjustable strabismus surgery under topical anaesthesia. Methods of local anaesthesia administration for strabismus surgery include retrobulbar, peribulbar, subtenons, and subconjunctival infiltration of local anaesthetic solution. ${ }^{29}$ However, when these methods are used with an adjustable suture technique, a delay in adjustment of at least $6 \mathrm{~h}$ is required to make sure the effects of the local anaesthetics have adequately dissipated before the adjustment can be performed. ${ }^{30,31}$ As no other method of anaesthesia was used in our study, the effect of ketorolac was more clearly assessed. 
The most frequently recorded pains began when the muscle was hooked for isolation and when the muscle was stretched during suturing before resecting the muscle. Ketorolac proved to be effective in decreasing the intraoperative pain with this procedure.

In a preliminary study, we injected intravenous ketorolac $(30 \mathrm{mg})$ with no definite effect. We hypothesised that the amount given was not sufficient to reduce intra- and postoperative pain; therefore, $60 \mathrm{mg}$ was administered in our follow-up study. The daily maximal intravenous administration dose for ketorolac tromethamine (Keromin; HanaPharm, Seoul, South Korea) recommended by the manufacturer was $90 \mathrm{mg}$. In cases for the elderly (over 65 years old), body weight under $50 \mathrm{~kg}$, and patients with renal insufficiency, the maximum daily dose should not exceed $60 \mathrm{mg}$. The maximal daily dose administered intravenously recommended by the First DataBank, ${ }^{32}$ which provides guidelines for our hospital, is $4 \mathrm{mg} / \mathrm{kg}$ per day for children from age 180 days to under 16 years old and $120 \mathrm{mg}$ /day for patients from 16 years old to under 65 years old. The mean age of ketorolac group was 26 years old (range, 11-61 years) and control group 20.1 years old (range, 11-54 years), and the mean body weight of ketorolac group was $59.97 \mathrm{~kg}$ (range, $45-100 \mathrm{~kg}$ ) (mean dose, $1.0 \mathrm{mg} / \mathrm{kg}$, range, $0.6-1.3 \mathrm{mg} / \mathrm{kg}$ ) and control group $61.33 \mathrm{~kg}$ (range, 35-75), so no patient exceeded their recommended maximal daily dose.

The adverse effects of ketorolac are gastrointestinal disturbances and renal impairment; however, the reported incidence is low and clinically insignificant. ${ }^{33-35}$ However, Strom et $a l^{35}$ reported that overall associations between ketorolac use and both gastrointestinal bleeding and operative site bleeding was small and the risk associated with the drug was larger and clinically important when ketorolac is used in larger doses ( $>105 \mathrm{mg} / \mathrm{d}$ ), in older subjects ( $>65$ years), and for more than 5 days. Although ketorolac is associated with a slightly higher incidence of postoperative bleeding $(0.4 \%)$ than not receiving the drug $(0.2 \%)$, it has not been reported to be a problem in strabismus surgery. ${ }^{11,12,28}$ Morrison et $a l^{12}$ reported intravenous ketorolac $60 \mathrm{mg}$ (mean dose $0.83 \mathrm{mg} / \mathrm{kg}$, range $0.61-1.08 \mathrm{mg} / \mathrm{kg}$ ) to be more effective than either oral acetaminophen $(650 \mathrm{mg})$ or oral ibuprofen $(600 \mathrm{mg})$ given 30-45 min after strabismus surgery in controlling postoperative pain without experiencing any episodes of remarkable bleeding or cutaneous bruising noted in the patients enroled in their study. Also Mui et al ${ }^{36}$ reported that no serious complication related to ketorolac was noted when $60 \mathrm{mg}$ (mean dose, $0.95 \mathrm{mg} / \mathrm{kg}$ ) was administered intravenously as premedication in colonoscopy.
In this study, there was no increase in perioperative bleeding in the ketorolac treatment group. In addition, no intraoperative complications such as the oculocardiac reflex, nausea, or vomiting occurred with the topical anaesthesia. The vital signs and consciousness level, skin tone, operation site bleeding, and any discomfort were checked and recorded by the nurses in the recovery room right after the surgery and before the patient was dismissed which was 3-5 h after surgery. Although all patients complained of fear and anxiety before surgery, none of the patients complained of gastrointestinal disturbances or oliguria or urinary retention after surgery nor was operation site bleeding recorded by the nurses. Also during the out-patient clinic follow-up, no operation site bleeding or periocular bruising was noted by the surgeon nor were gastrointestinal disturbances, renal complications, skin rashes, and allergic symptoms complained by the patient. Although we did no follow-up laboratory tests after using ketorolac, the monitoring recommended by the manufacturer is complete blood cell count, chemistry profile, blood pressure, fecal occult blood test, and liver function after initiation of NSAID therapy and every 6-12 months thereafter if prescribed for longer than the recommended 5 days. More frequent monitoring is recommended in patients at risk for hepatic, renal, cardiac, or gastrointestinal toxicity (eg, age greater than 60 years, high-dose NSAID therapy, concurrent corticosteroids or anticoagulants, history of cardiac, renal, or hepatic disease). Bleeding time monitoring is recommended in patients with coagulation disorders or on anticoagulants and renal function in patients with pre-existing renal dysfunction. As none of our patients fell into these categories nor complained of side effects, we felt no need to perform further postoperative laboratory tests. Despite the adverse effects of ketorolac, we believe that it is uncommon to experience clinically severe side effects after a single dose of intravenous ketorolac in healthy subjects before strabismus surgery.

The reasons we think that the patient satisfaction was similar in both groups were mainly because of the skill of the surgeon and patients anticipation. The surgeon had performed more than 10000 cases of strabismus surgeries and 1000 cases under topical anaesthesia with adjustable sutures, so minimal suffering was experienced by the patients compared with being operated by an unskilful surgeon. Also, as we reviewed the medical records of the recovery room nurses, we found that the fear and anxiety the patients complained before the surgery was much more than we thought. Every patient was frightened that surgery would be done only under topical anaesthesia and was worried what would happen to them if their eyes would not be able to keep still during surgery. So we think that the thought of the 
surgery being over itself might have made the patients to be satisfied in both groups regardless of the difference in pain.

In conclusion, ketorolac tromethamine appears to be an effective non-narcotic alternative for pain control in patients undergoing single-stage adjustable strabismus surgery. Ketorolac decreases eye pain intraoperatively and in the immediate postoperative period compared with those of the saline placebo. Expeditious hospital discharge because of less pain and shorter adjustment time with single-stage adjustable strabismus surgery has advantages in terms of patient stress, satisfaction, and health-care cost efficacy. Finally, when patients experience less pain, cooperation can improve, allowing adjustable strabismus surgery to become a more attractive procedure for both patients and surgeons.

\section{Summary}

\section{What was known before}

- The effect of ketorolac has been evaluated in strabismus surgery; however, in these studies the patients were under general or periocular anaesthesia.

\section{What this study adds}

- We prospectively evaluated that intravenous ketorolac, when administered preoperatively for single-stage adjustable strabismus surgery under topical anaesthesia, was effective in reducing pain during and after surgery.

\section{Conflict of interest}

The authors declare no conflict of interest.

\section{References}

1 Thorson JC, Jampolsky A, Scott AB. Topical anesthesia for strabismus surgery. Trans - Am Acad Ophthalmol Otolaryngol 1966; 70(6): 968-972.

2 Buffenn AN. Adjustable suture use in strabismus surgery. Curr Opin Ophthalmol 2005; 16(5): 294-297.

3 Koc F, Durlu N, Ozal H, Yasar H, Firat E. Single-stage adjustable strabismus surgery under topical anesthesia and propofol. Strabismus 2005; 13(4): 157-161.

4 Ward JB, Niffenegger AS, Lavin CW, Acquadro MA, Ahern DK, Smith PV et al. The use of propofol and mivacurium anesthetic technique for the immediate postoperative adjustment of sutures in strabismus surgery. Ophthalmology 1995; 102(1): 122-128.

5 Chang Y, Ryu IH, Han S, Lee S, Lee J. Intraoperative adjustment in strabismus surgery under topical anesthesia. Yonsei Med J 2006; 47(5): 667-671.

6 Chow PC. Stability of one-stage adjustable suture for the correction of horizontal strabismus. Br J Ophthalmol 1989; 73(7): 541-546.

7 Rooks II WH, Tomolonis AJ, Maloney PJ, Wallach MB, Schuler ME. The analgesic and anti-inflammatory profile of ( + /-)-5-benzoyl-1,2-dihydro-3H-pyrrolo[1,2a]pyrrole1-carboxylic acid (RS-37619). Agents and Actions 1982; 12(5-6): 684-690.

8 Bravo LJ, Mattie H, Spierdijk J, Bovill JG, Burm AG. The effects on ventilation of ketorolac in comparison with morphine. Eur J Clin Pharmacol 1988; 35(5): 491-494.

9 Gillies GW, Kenny GN, Bullingham RE, McArdle CS. The morphine sparing effect of ketorolac tromethamine. A study of a new, parenteral non-steroidal anti-inflammatory agent after abdominal surgery. Anaesthesia 1987; 42(7): 727-731.

10 Watcha MF, Jones MB, Lagueruela RG, Schweiger C, White PF. Comparison of ketorolac and morphine as adjuvants during pediatric surgery. Anesthesiology 1992; 76(3): 368-372.

11 Mendel HG, Guarnieri KM, Sundt LM, Torjman MC. The effects of ketorolac and fentanyl on postoperative vomiting and analgesic requirements in children undergoing strabismus surgery. Anesth Analg 1995; 80(6): 1129-1133.

12 Morrison NA, Repka MX. Ketorolac vs acetaminophen or ibuprofen in controlling postoperative pain in patients with strabismus. Ophthalmology 1994; 101(5): 915-918.

13 Munro HM, Riegger LQ, Reynolds PI, Wilton NC, Lewis IH. Comparison of the analgesic and emetic properties of ketorolac and morphine for paediatric outpatient strabismus surgery. Br J Anaesth 1994; 72(6): 624-628.

14 Wright KW. Color Atlas of Strabismus Surgery: Strategies and Techniques. Springer: New York, 2007.

15 Sharma P, Julka A, Gadia R, Chhabra A, Dehran M. Evaluation of single-stage adjustable strabismus surgery under conscious sedation. Indian J Ophthalmol 2009; 57(2): 121-125.

16 Eustis HS, Eiswirth CC, Smith DR. Vagal responses to adjustable sutures in strabismus correction. Am J Ophthalmol 1992; 114(3): 307-310.

17 Jampolsky A. Current techniques of adjustable strabismus surgery. Am J Ophthalmol 1979; 88(3): 406-418.

18 Kim S, Yang Y, Kim J. Tolerance of patients and postoperative results: topical anesthesia for strabismus surgery. J Pediatr Ophthalmol Strabismus 2000; 37(6): 344-348.

19 Melhuish JA, Kemp EG. The routine use of adjustable sutures in adult strabismus surgery. J R Coll Surg Edinb 1993; 38(3): 134-137.

20 Rosenbaum AL, Metz HS, Carlson M, Jampolsky AJ. Adjustable rectus muscle recession surgery. A follow-up study. Arch Ophthal 1977; 95(5): 817-820.

21 Wygnanski-Jaffe T, Wysanbeek Y, Bessler E, Spierer A. Strabismus surgery using the adjustable suture technique. J Pediatr Ophthalmol Strabismus 1999; 36(4): 184-188.

22 Seijas O, de Liao PG, Merino P, Roberts CJ, de Liao RG. Topical anesthesia in strabismus surgery: a review of 101 cases. JPediatr Ophthalmol Strabismus 2009; 46(4): 218-222.

23 Fekrat S, Marsh MJ, Elsing SH, Raja SC, de Juan Jr E, Campochiaro PA et al. Intraoperative ketorolac and eye pain after viteoretinal surgery: a prospective, randomized, placebo-controlled study. Retina 2003; 23(1): 8-13.

24 Brown CR, Moodie JE, Wild VM, Bynum LJ. Comparison of intravenous ketorolac tromethamine and morphine sulfate in the treatment of postoperative pain. Pharmacotherapy 1990; 10(6 (Part 2)): 116S-121S.

25 Buckley MM, Brogden RN. Ketorolac. A review of its pharmacodynamic and pharmacokinetic properties, and therapeutic potential. Drugs 1990; 39(1): 86-109.

26 O'Hara DA, Fragen RJ, Kinzer M, Pemberton D. Ketorolac tromethamine as compared with morphine sulfate for 
treatment of postoperative pain. Clin Pharmacol Ther 1987; 41(5): 556-561.

27 Shende D, Das K. Comparative effects of intravenous ketorolac and pethidine on perioperative analgesia and postoperative nausea and vomiting (PONV) for paediatric strabismus surgery. Acta anaesthesiologica Scandinavica 1999; 43(3): 265-269.

28 Williams AR, Conroy JM. The anesthetic management of the pediatric strabismus patient. J AAPOS 1998; 2(2): 113-115.

29 Hakim OM, El-Hag YG, Haikal MA. Strabismus surgery under augmented topical anesthesia. J AAPOS 2005; 9(3): 279-284.

30 Guyton DL. A testimony for Postop' adjustable sutures in strabismus surgery, on the 25th (Silver) anniversary of Arthur Jampolsky's original description. Binocul Vis Strabismus Q 2001; 16(4): 241-242.

31 Velez FG, Chan TK, Vives T, Chou T, Clark RA, Keyes $\mathrm{M}$ et al. Timing of postoperative adjustment in adjustable suture strabismus surgery. I AAPOS 2001; 5(3): 178-183.

32 FirstDataBank. http://www.firstdatabank.com. Hearst Corporation.

33 Sinha V, Kumar R, Singh G. Ketorolac tromethamine formulations: an overview. Expert Opin Drug Deliv 2009; 6(9): 961-975.

34 Oliva A, De Giorgio F, Arena V, Fucci N, Pascali VL, Navarra P. Death due to anaphylactic shock secondary to intravenous self-injection of Toradol: a case report and review of the literature. Clin Toxicol 2007; 45(6): 709-713.

35 Strom BL, Berlin JA, Kinman JL et al. Parenteral ketorolac and risk of gastrointestinal and operative site bleeding. A postmarketing surveillance study. JAMA 1996; 275(5): 376-382.

36 Mui WL, Kwong W, Li AC et al. Premedication with intravenous ketorolac trometamol (Toradol) in colonoscopy: a randomized controlled trial. Am J Gastroenterol 2005; 100(12): 2669-2673. 